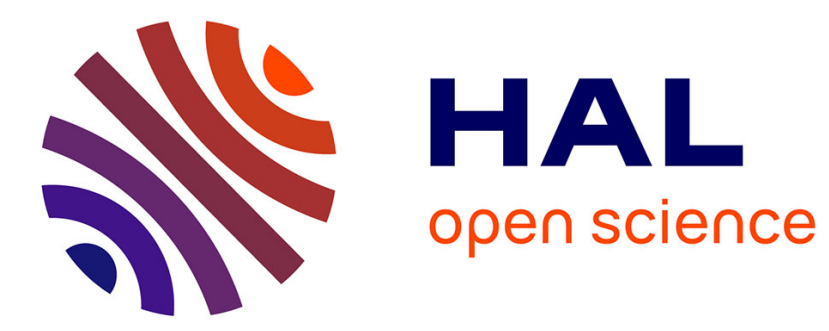

\title{
On the Uncertainty of CFD in Sail Aerodynamics
}

\author{
I.M Viola, Patrick Bot, M. Riotte
}

\section{To cite this version:}

I.M Viola, Patrick Bot, M. Riotte. On the Uncertainty of CFD in Sail Aerodynamics. International Journal for Numerical Methods in Fluids, 2013, 72 (11), pp.1146-1164. 10.1002/fld.3780 . hal01071699

\section{HAL Id: hal-01071699 \\ https://hal.science/hal-01071699}

Submitted on 6 Oct 2014

HAL is a multi-disciplinary open access archive for the deposit and dissemination of scientific research documents, whether they are published or not. The documents may come from teaching and research institutions in France or abroad, or from public or private research centers.
L'archive ouverte pluridisciplinaire HAL, est destinée au dépôt et à la diffusion de documents scientifiques de niveau recherche, publiés ou non, émanant des établissements d'enseignement et de recherche français ou étrangers, des laboratoires publics ou privés. 


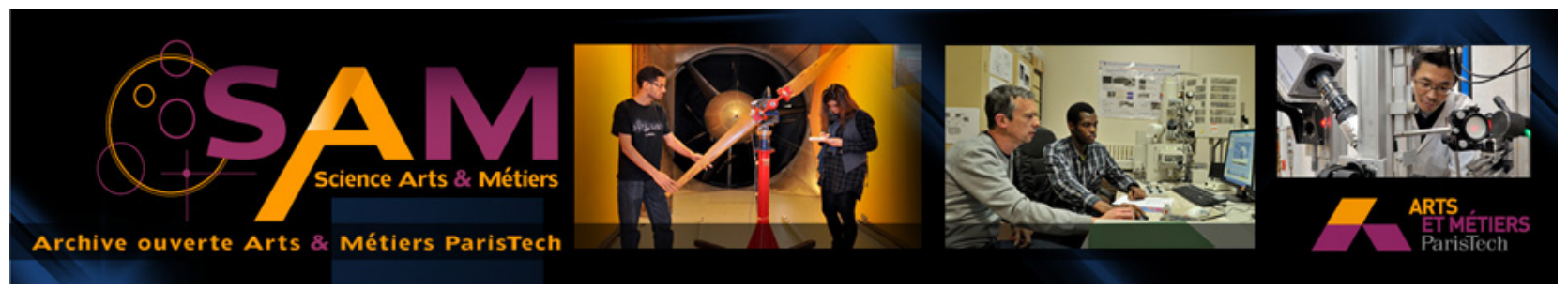

Science Arts \& Métiers (SAM)

is an open access repository that collects the work of Arts et Métiers ParisTech researchers and makes it freely available over the web where possible.

This is an author-deposited version published in: http://sam.ensam.eu Handle ID: .http://hdl.handle.net/10985/8697

\section{To cite this version :}

I.M VIOLA, Patrick BOT, M. RIOTTE - On the Uncertainty of CFD in Sail Aerodynamics International Journal for Numerical Methods in Fluids - Vol. 72, n¹1, p.1146-1164 - 2013 


\author{
Viola, I.M., Bot, P, Riotte, M \\ On the Uncertainty of CFD in Sail Aerodynamics
}

\title{
Keywords
}

Sail Aerodynamics; Yacht; Computational Fluid Dynamics (CFD); Raynoldsaveraged Navier -Stokes (RANS); Verification and Validation (V\&V)

\begin{abstract}
A verification and validation procedure for yacht sail aerodynamics is presented. Guidelines and an example of application are provided. The grid uncertainty for the aerodynamic lift, drag and pressure distributions for the sails is computed. The pressures are validated against experimental measurements, showing that the validation procedure may allow the identification of modelling errors. Lift, drag and $L 2$ norm of the pressures were computed with uncertainties of the order of $1 \%$. Convergence uncertainty and round-off uncertainty are several orders of magnitude smaller than the grid uncertainty. The uncertainty due to the dimension of the computational domain is computed for a flat plate at incidence and is found to be significant compared with the other uncertainties. Finally, it is shown how the probability that the ranking between different geometries is correct can be estimated knowing the uncertainty in the computation of the value used to rank.
\end{abstract}

\section{Introduction}

\subsection{Background}

The aerodynamics of sailing vessels has been studied for centuries, since the days when sails were primarily used by merchant fleets. Only since the middle of the nineteenth century, with the birth of modern yachting, has sail aerodynamics been associated with recreational sailing boats. Today, this branch of fluid dynamics is of increasing interest both due to the impact of sailing competitions on the economy of some countries, and to the possible future applications of sails for renewable wind energy devices. In fact, the most important sailing competition, the America's Cup, today generates revenues that are only $20 \%$ lower than those generated by the FIFA World Cup [1]. Since the 1990s, the most competitive America's Cup sailing teams have each invested around $£ 20$ million every year in order to achieve the fastest possible yacht, and these figures are likely to increase further in future years. In addition, due to the constant increase in fuel and oil costs and the unsustainable production of carbon emissions by current merchant fleets, sail propulsion is again becoming an attractive alternative for the future.

An increasing number of scientists and resources are being employed to study the performance of sails. The constant growth of computational capabilities makes computational fluid dynamics (CFD) the most used design tool in sail aerodynamics today. The increase in number of numerical simulations performed requires guidelines and good practice to be established to support inexperienced users and industrial applications in the sail aerodynamic field. In particular, verification and validation 
(V\&V) analysis would allow scientists to assess and enhance simulations, and sail and yacht designers to make better use of the results.

\subsection{Verification and Validation}

The aim of the $\mathrm{V} \& \mathrm{~V}$ procedure is to assess the error computed by numerical simulations. In fact, performing a numerical simulation implies a simplification of reality through the use of a model, and the discretisation of the continuum by a finite number of elements, time steps, iterations, digits, etc. These two simplifications lead to differences, namely modelling and numerical errors, between the real value and the computed value. V\&V aims to assess the magnitude and the sign of the modelling and numerical errors. In the last ten years several methodologies have been presented, mainly applied to the aeronautical fields. Whereas in other fields, such as hull hydrodynamics [2], a formal V\&V procedure is regularly adopted and workshops are held to test different approaches and methodologies [3-8] in sail aerodynamics a formal $V \& V$ procedure has never been presented before. The aim of the present paper is to show an example of $V \& V$ applied to sail aerodynamics that can be adopted as a guideline for this field of application. The most acknowledged V\&V procedures are reviewed. In particular, methods based on the Grid Convergence Index [9-11], the Factor of Safety [12-14] and the Least Square Root [15-18] are considered. These methods were used to draw the guidelines proposed by the American Society of Mechanical Engineers (ASME) [19] and by the CFD Specialist Committee of the International Towing Tank Conference (ITTC) [20-22]. Unfortunately, these methods are complicated to implement and have different limitations. In particular, the Grid Convergence Method does not take into account the scatter in the numerical results, while the Factor of Safety and the Least Square Root methods require knowledge of the theoretical order of convergence, which is often unknown when commercial codes are used and different orders are exploited for different equations. Therefore, a simplified approach, mainly inspired from [17,18] and which can be easily implemented in industrial practice where tight timeframes do not allow a large number of simulations to be performed, is suggested herein.

\section{Presentation of the $\mathrm{V} \& \mathrm{~V}$ procedure}

The V\&V aims to estimate the error in the computation of any primitive, derivative, local and global variables. For any of these variables, indicated with $\phi$ herein, the error $\delta_{\phi}$ is defined as the difference between the computed value $\phi_{C F D}$ and the true value $\phi_{T R U E}$ :

$$
\delta_{\phi}=\phi_{C F D}-\phi_{T R U E}
$$

The use of the subscript $\phi$ in Equation 1 and in the following Equations remarks that the presented V\&V procedure should be repeated for every variable $\phi$ of interest. The error $\delta_{\phi}$ is broken down into two components: the modelling error $\delta_{\phi_{\text {mod }}}$, which is due to the simplification of the model, and the numerical error $\delta_{\phi_{n u m}}$, which is due to the difference between the continuous nature of the reality and the discrete nature of the numerical model.

$$
\delta_{\phi}=\delta_{\phi_{\text {mod }}}+\delta_{\phi_{\text {num }}}
$$


In some $\mathrm{V} \& \mathrm{~V}$ procedures, such as those recommended by ASME [19], a third error component due to the uncertainty of the simulation's inputs is considered. Conversely, in the proposed procedures, in agreement with the ITTC guidelines [20-22], this component is included in the numerical error.

In sail aerodynamics, the modelling error can be due, for instance, to the neglected fluid-structure interaction when the fluid dynamic computation is not coupled with a structural code, to the neglected dynamic movements of the yacht that leads to a variation of the experienced wind speed and direction, or to the modelling of the atmospheric boundary layer when the measurement of the vertical velocity profile is not available. When wind tunnel tests instead of real sailing conditions are modelled, modelling errors can be due, for instance, to the position of the external boundaries. In fact, the wind tunnel walls lead to a blockage effect, which affects both the velocity magnitude and the direction of the onset flow. Other sources of modelling errors are the geometry of the yacht model, the rigging and the thickness of the sails, the turbulence model, the near wall treatment, etc.

The numerical error $\left(\delta_{\phi_{\text {num }}}\right)$ is a function of errors due to the grid $\left(\delta_{\phi_{g}}\right)$, time step $\left(\delta_{\phi_{t}}\right)$, round-off $\left(\delta_{\phi_{r}}\right)$, other input parameters $\left(\delta_{\phi_{p}}\right)$ and convergence $\left(\delta_{\phi_{c}}\right)$.

$$
\delta_{\phi_{\text {num }}}=f\left(\delta_{\phi_{g}}, \delta_{\phi_{t}}, \delta_{\phi_{r}}, \delta_{\phi_{p}}, \delta_{\phi_{c}}\right)
$$

While the true value $\phi_{T R U E}$ or its experimental estimate must be available to assess the modelling error, the numerical error is assessed without any information about $\phi_{T R U E}$. In fact, because the numerical error is due to the discrete nature of the numerical model, it is possible to assess this error by testing different discretisations of the model. This is a very important point that is worth highlighting. Therefore, even if the experimental data is not available, the uncertainty of the computed results can still be estimated. This uncertainty will include the contribution due to the numerical error and not the one due to the modelling error.

The process that assesses the numerical error and the associated numerical uncertainty, $U_{\phi_{\text {num }}}$, is called verification. In particular, it assesses uncertainty components due to the grid size $\left(U_{\phi_{g}}\right)$, time step $\left(U_{\phi_{t}}\right)$, round-off $\left(U_{\phi_{r}}\right)$ other input parameters $\left(U_{\phi_{p}}\right)$ and convergence $\left(U_{\phi_{c}}\right)$. The overall numerical uncertainty is estimated as per experimental fluid dynamics uncertainty analysis, shown in Equation 4.

$$
U_{\phi_{\text {num }}}=\sqrt{U_{\phi_{g}^{2}}^{2}+U_{\phi_{t}^{2}}^{2}+U_{\phi_{r}}^{2}+U_{\phi_{p}^{2}}^{2}}+U_{\phi_{c}}
$$

The discretisation uncertainty is not independent from the convergence uncertainty [23] and for this reason it is linearly added in Equation (4).

The following sections present how each of the uncertainties on the right hand side of Equation 4 can be assessed. The uncertainties are provided at $95 \%$ confidence levels, 
assuming a normal distribution of the errors. Therefore, assuming normally distributed errors, the uncertainty is defined as two standard deviations of the error distribution and the solution $\phi$ has probabilities of $68.3 \%, 95.4 \%$ and $99.7 \%$ to lay within the intervals $\left[\phi-\frac{U_{\phi}}{2}, \phi+\frac{U_{\phi}}{2}\right],\left[\phi-U_{\phi}, \phi+U_{\phi}\right]$ and $\left[\phi-\frac{3}{2} U_{\phi}, \phi+\frac{3}{2} U_{\phi}\right]$ respectively. Figure 1 shows the probability distribution of $\phi$. It should be noted that the error bars used herein do not represent the maximum variation of $\phi$. Conversely, there is a probability of $2.3 \%$ that $\phi$ is higher, and $2.3 \%$ that $\phi$ is smaller, than the maximum and minimum extremes of the error bars respectively.

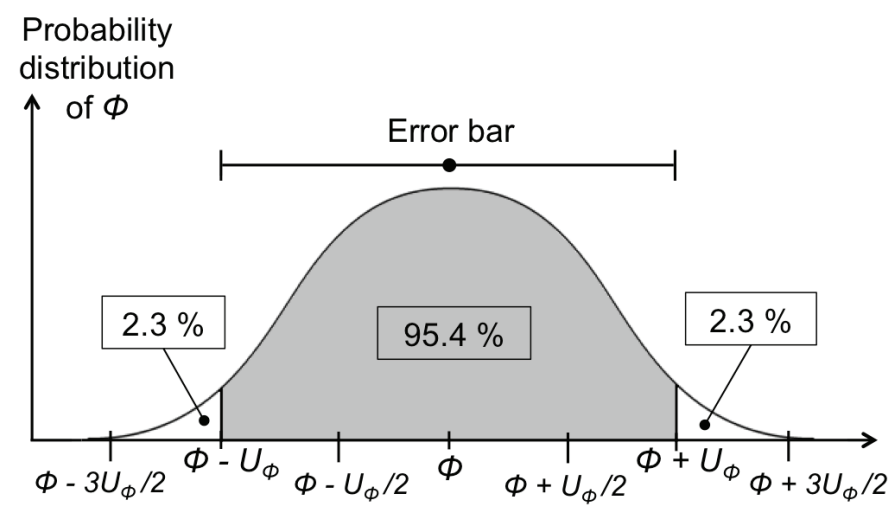

Figure 1: Probability distribution of $\phi$ and definition of the error bar.

\subsection{Discretisation Uncertainties}

When Reynolds-averaged Navier-Stokes equations (RANSE) are solved, the space and time resolutions set the limit between the physics resolved by the simulation and that modelled with semi-empirical formulations. Therefore, varying the resolution leads to different results. When very poor resolution is used, a variation in the resolution can lead to an unpredictable jump of the solution, and increasing the resolution does not lead to a converging solution. This range of resolutions is called the stochastic range. When the resolution is able to model most of the flow features that affect the solution, then increasing the resolution leads to an asymptotically converging solution. This range of resolution is called the asymptotic range. The convergence can be monotonic or oscillatory. The amplitude of the oscillations can be partially controlled with the under-relaxation factors, although a scatter can be superimposed on the asymptotic trend. This can be due to various sources, for instance, the switch between two different model formulations in the near-wall region triggered by the value of the wall distance in boundary layer units $y^{+}$. Distinguishing between a non-converging trend and a converging trend affected by the scatter can be very difficult and examples will be provided in the following sections. Figure 2 shows the stochastic and the asymptotic ranges for a generic value $\phi$. The time and space discretisation resolution is represented by the step size $h$, which is the time step for the time resolution, and a characteristic distance between two grid points for the space resolution. The step size is divided by a base step size $h_{\text {base }}$, which is used as a reference. Figure 2 shows that when the step size decreases, the solution varies randomly (stochastic range) until it converges asymptotically towards $\phi_{0}$ (asymptotic range). 


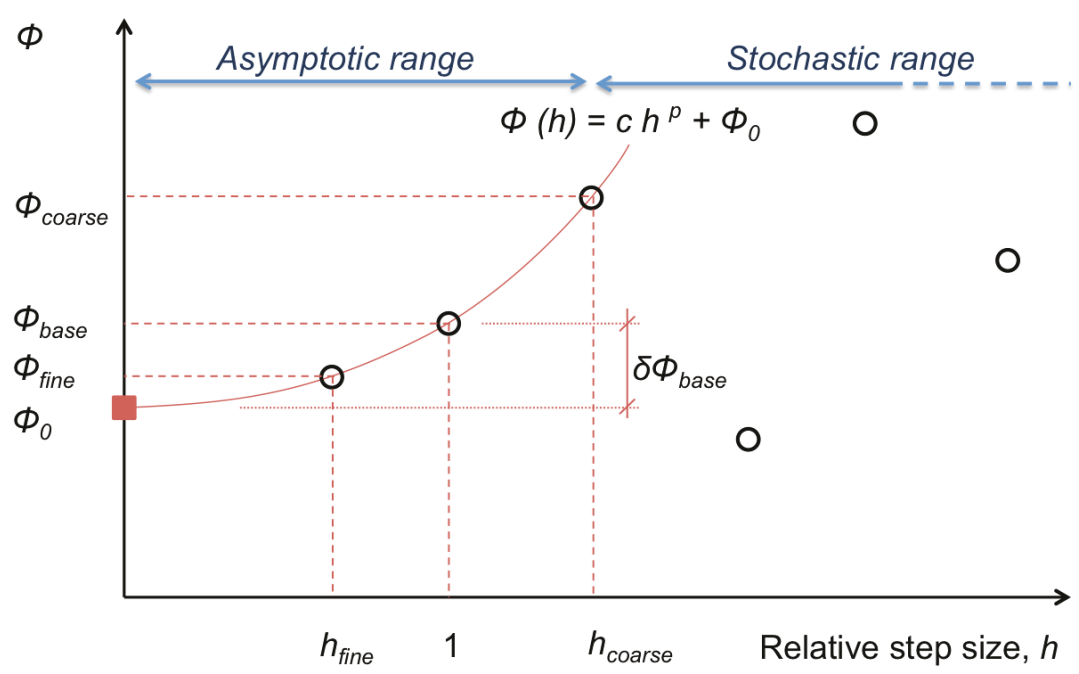

Figure 2: Asymptotic range for a solution $\phi$ versus the relative step size $h$.

A numerical code should converge towards a grid independent solution with the same order of convergence as the order of accuracy of the discretisation scheme adopted. For instance, a steady diffusion-convective problem solved on a regular grid should converge with first, second and third orders when the convective term is modelled with an upwind differencing scheme (UDS), a central differencing scheme (CDS) or a quadratic upstream interpolation for convective kinetics (QUICK) respectively [24]. In fact, these three schemes are first, second and third order accurate on regular grids. In other words, if the grid spacing between the nodes were decreased by $X \%$, the numerical error due to the grid resolution would decrease by $X \%,(X \%)^{2}$ and $(X \%)^{3}$ respectively. Similarly, a time-dependent diffusive only problem should present a first and second order convergence when solved with an Euler (implicit or explicit) and a Crank-Nicholson method, respectively. Unfortunately, in sail aerodynamics different schemes with different orders of accuracy are used at the same time and the grids are non uniform, therefore, the order of convergence is not predictable and should be assessed. The order of convergence allows the uncertainty of the computed value to be assessed.

The converging trend $\phi=\phi(h)$ can be expanded in a power series in order to compute the order of convergence and the extrapolated value $\phi_{0}$ for $h=0$. When three equidistant step sizes are available, then the Richardson Extrapolation can be used $[9,10,25]$.

Hereby, a more general formulation, which allows estimating the uncertainty at a $95 \%$ confidence level and which can be used with any number of step sizes above two and with any relative distance, is presented. The convergence trend is approximated in Equation 5, and the coefficients $c, p$ and $\phi_{0}$ are computed with the least-squares method. In addition, the standard deviation $\sigma$ of the fit must be computed.

$$
\phi(h)=c h^{p}+\phi_{0}
$$

If $p \geq 0.95$, the uncertainty in the computation of $\phi$ due to a step size $h$ is computed using Equation 6. 
where

$$
U_{\phi_{h}}=1.25\left|\delta_{\phi_{h}}\right|+\sigma
$$

$$
\delta_{\phi_{h}}=\phi_{h}-\phi_{0}
$$

The subscript $h$ in Equation 6 is to remind that this is the uncertainty for the step size $h$, which can be either the grid step size or the time step size; the value 1.25 is a safety factor, and the vertical bars in Equation 7 stand for the absolute value of the argument between the bars. The higher the order of convergence, $p$, the smaller $\left|\delta_{\phi_{h}}\right|$ and, thus, the uncertainty $U_{\phi_{h}}$. Unrecognised scatter might lead to an incorrect estimate of the order of convergence. In particular, when different algorithms with orders of accuracy between one and three are used, the orders of convergence below one and above three are unlikely and additional simulations with different step sizes are recommended, though it should be taken into account that the use of limiters might lead to orders below one. Additional simulations may allow a better estimation of the order of convergence. The standard deviation, $\sigma$, of the least-squares method introduces an additional uncertainty in the measurement of the order of convergence. For this reason, the larger the standard deviation, $\sigma$, the larger the uncertainty. It should be noted that when only three step sizes are used, then the three coefficients $c, p$ and $\phi_{0}$ can be computed exactly and $\sigma=0$. The limitation of Equation 6 is that testing a lower number of step sizes might lead to a lower uncertainty, which is not justified. This is particularly relevant for low orders of convergence and high scatter. Therefore, a different equation is suggested for $p<0.95$.

Equations 6-7 suggest a formulation non dissimilar from the one proposed by [17]. However, these authors assume a theoretical order of accuracy of 2 . Therefore, if $p \geq 2.05$, they recommend verifying if a larger uncertainty is computed using $p=2$ for the fit and a safety factor of 3 .

If $p<0.95$, the uncertainty in the computation of $\phi$ due to any step size $h$ is computed using Equation 8, where a safety factor of 1.5 is used.

where

$$
U_{\phi_{h}}=1.5 \delta_{\Delta_{\phi}}+\sigma
$$

$$
\delta_{\Delta_{\phi}}=\frac{\phi_{\max }-\phi_{\min }}{1-\frac{h_{\min }}{h_{\max }}}
$$

$\delta_{\Delta_{\phi}}$ is equal to the data range $\left(\phi_{\max }-\phi_{\min }\right)$ scaled by the ratio between the amplitude of the relative step size range $\left(h_{\max }-h_{\min }\right)$ and its distance from the $h=0$ axis $\left(h_{\max }-0\right)$. This scaling function allows the limitation of Equation 6 , as discussed above, to be partially overcome. In fact, if the range of relative steps is increased, for instance, the uncertainty decreases.

This novel correction is inspired by the formulation proposed by [17], where the authors corrected the data range with a function of the relative step size range and, as in this formulation, when $h_{\max }=2$ and $h_{\min }=1$, then $U_{\phi_{h}}=3\left(\phi_{\max }-\phi_{\min }\right)$. 
This concludes the uncertainty analysis due to the space and time discretisation. The reader can also find a similar guideline in the Appendix of [26], where the authors present a detailed analysis of the convergent trends of the friction and pressure resistance components of a tanker for several grid resolutions.

It is common practice to assess the uncertainty for one condition (for instance one sail trim) and then model different conditions (for instance different sail trims) assuming that the uncertainty is independent from the modelled condition. For all the other conditions, only one value of $\phi$ is computed, and the uncertainty $U_{\phi}$ computed on a different condition is assumed to be valid. This is an acceptable but dangerous assumption. For this reason, Equation 8 is a conservative approach to compute the uncertainty. For the condition that was used for the uncertainty assessment, where more grid and time step sizes are computed, it is possible to compute a more accurate value of $\phi$ than $\phi_{h}$. In particular, if $p \geq 0.95$, then $\phi_{0}$ could be a better approximation than $\phi_{h}$. However, the computed uncertainty is valid for $\phi_{h}$ and not for $\phi_{0}$, and using $\phi_{0}$ is not recommendable because unrecognised scatter might lead to a large error in the estimation of $\phi_{0}$.

If $-0.05 \leq p \leq 0.05$ and a $N$ step sizes are used, in order to provide a more accurate estimation of $\phi$, the mean value $\phi_{\text {mean }}$ computed with different step sizes can be used. The uncertainty in the computation of $\phi_{\text {mean }}$ can be computed with Equation 10, where $\sigma_{\phi}$ is the standard deviation of the distribution of $\phi$ :

$$
U_{\phi_{\text {mean }}}=2 \frac{\sigma_{\phi}}{\sqrt{N}}
$$

\subsection{Convergence Uncertainty}

The uncertainty correlated with the convergence criterion is also known as iterative uncertainty. This is due to the difference between the solution converged to a given convergence criterion and the solution converged to machine accuracy, meaning to the maximum number of digits used to store the solution. In most cases, convergence to machine accuracy cannot be achieved and the convergence to a much less demanding criterion might lead to a negligible uncertainty. Therefore, in order to minimise the computational time, the convergence criterion should be chosen in order to be negligible compared with uncertainties due to other sources, such as the discretisation.

The convergence error can be estimated from the normalised residuals of the continuity and momentum equations, or from the changes in a local or global quantity. [23] showed that these two options are equivalent when the $L_{\infty}$ norm $\left(\phi_{\max }-\phi_{\min }\right)$ on the complete flow field is used. They also showed that using only the results of the last two iterations can lead to under-estimation of the convergence uncertainty by up to one order of magnitude.

A simple way to assess the convergence uncertainty of $\phi$ is to use its convergence history. For instance, if $\phi$ is the lift generated by the sails, this value can be monitored for every iteration and the uncertainty can be computed from the analysis of the trend of the lift versus the iteration advancement. The data can be fitted with Equation 11 
by the least-squares method, where $x_{n}$ is an opportune variable proportional to the iteration number $\mathrm{n}$ (for instance, $x_{n}=n$ ), and $\phi_{\infty}$ is the extrapolated value for an infinite number of iterations. The fit will lead to a negative value of $p$.

$$
\phi\left(x_{n}\right)=c x_{n}^{p}+\phi_{\infty}
$$

The extrapolated value $\phi_{\infty}$ is the best estimation of $\phi$, and the uncertainty can be computed with Equation 12, where $\sigma$ is the standard deviation of the fit and $\phi_{c}$ is the value computed at the last iteration.

where

$$
U_{\phi_{c}}=1.25\left|\delta_{\phi_{c}}\right|+\sigma
$$

$$
\delta_{\phi_{c}}=\phi_{c}-\phi_{\infty}
$$

The initial iterations, when large-amplitude oscillations of $\phi$ can be computed, should be excluded from the fit, otherwise this method can lead to too conservative an estimate of the iterative error.

\subsection{Round-Off Uncertainty}

The round-off error, also known as the rounding error, is the difference between the number stored in the memory of the machine and its exact mathematical value. For instance, for a simulation performed in double precision on a 32-bit machine, the used number of digits is 15. Although this error is normally very small, it can be significantly magnified through successive operations, and particularly when computing the difference between quantities close to each other. In order to assess the uncertainty due to the round-off, different accuracies at which the numbers are stored should be compared. For instance, simulations can be computed on 16-, 32- and 64bit machines. In practice, most CFD software allows the simulations to be run in single or double precision. Therefore, it is good practice to verify that the difference between the solution achieved with single precision, $\phi_{s p}$, and the solution achieved with double precision, $\phi_{d p}$, is negligible compared with the other errors mentioned above. The uncertainty due to the round-off can be computed using Equation 14.

where

$$
U_{\phi_{r}}=3 \delta_{r}
$$

$$
\delta_{r}=\left|\phi_{s p}-\phi_{d p}\right|
$$

Additional considerations on errors can be found in [23]. 


\subsection{Other Input Parameters Uncertainty}

Uncertainties due to many other parameters should be assessed but, in practice, only a few parameters are normally added to the list of sources of uncertainties. In fact, most other sources of errors, such as the two examples provided in this section, can be considered sources of modelling errors.

The methodology to assess the uncertainty due to any parameter should be considered on a case by case basis. When a superior or inferior limit of one parameter is associated with the exact solution, then the convergence of the solution, when the parameter tends towards that end, should be considered. For instance, in sail aerodynamics, when the far field wind velocity is known, the distance between the inlet boundary and the yacht is one parameter of this species. By increasing this distance, $d$, the solution $\phi=\phi(d)$ should converge towards a value independent of $d$. In this case a formulation analogous to that used for the step size (Section 2.1) can be used to assess the uncertainty, $U_{\phi_{d}}$, due to the parameter $d$, where the uncertainty is proportional to the difference between the extrapolated solution, $\phi(d \rightarrow \infty)$, and the solution $\phi(d)$ achieved with a given value of $d$.

When any limit of the parameter cannot be associated with the exact solution, then a more cautious approach should be used. For instance, if different turbulence models lead to different solutions, then the uncertainty can be estimated with a formulation analogous to that used for the round off (Section 2.3), where $\phi_{s p}$ and $\phi_{d p}$ in Equation 15 can be substitute with the maximum and the minimum values of the solutions achieved with different turbulence models, respectively.

\subsection{Experimental Uncertainty}

The previous sections (Sections 2.1-2.4) allow computation of the various components of the numerical uncertainties, which are then combined using Equation 4. The uncertainty in the computation of $\phi$ is due to both the numerical uncertainty and the modelling uncertainty. To assess this second component, the computed solution must be compared with the true solution. In sail aerodynamics, as in most applications, the true solution is unknown but experimental data might be available. In this case, the experimental uncertainty must be assessed.

A rigorous design and use of the measurement system allows the experimental error to be reduced and the associated uncertainty to be determined [27-28]. The experimental error for a generic quantity $\phi$ is defined as the difference between the measured value and the true value:

$$
\delta_{\phi_{\text {exp }}}=\phi_{E X P}-\phi_{T R U E}
$$

The first source of errors is due to the sensors used for the experimental measurements. For a given sensor, the so-called systematic error consists of the difference between the true value to be measured and the value given by the sensor which is constant or has slow variation compared with the measurement duration. The systematic error may be due to a sensor calibration error (offset or sensitivity), to wear of the sensor, to an incomplete knowledge of the experimental system or to a 
bias in using the sensor: for example, non-adapted sensor response time or perturbation of the measured value by the presence of the sensor. A systematic error results in a constant shift between the true value $\phi_{T R U E}$ and the measured value $\phi_{E X P}$. This may be estimated as the difference between the most probable values issued from several measurements of the same true value achieved with different methods and sensors. A sensor is said to be accurate if the systematic error is small.

Another source of experimental errors are the accidental errors, which are generally considered random and not correlated with each other. Random errors have numerous different origins listed below, intrinsic to the sensor or due to external causes. A random error is, for instance, the linearity error $\delta_{l}$ due to the linear assumption of the sensor response. The linearity error must be determined during the sensor calibration. The resolution error $\delta_{r}$ results from the mobility error $\delta_{m}$ due to the smallest variation of the true value detectable by the sensor and the possible reading error $\delta_{r d}$. The hysteresis error $\delta_{h}$ is defined as half the maximum difference in the measurement obtained for increasing and decreasing values. The quantification error $\delta_{q}$ due to an analogue-digital converter is defined as half the value of the least significant bit (LSB). A parasitic error is due to the internal noise or drift into the sensor, to parasitic interferences or to fluctuations in the power supply. The fluctuations of quantities affecting the true value or the sensor's characteristics (e.g. the temperature) which are not considered in the calibration lead to a systematic error if the measurement is carried out with a duration much shorter than the fluctuation time-scale or a random error if it is carried out with a much longer duration.

Random errors lead to a dispersion of the measured values for repeated measurements. A statistical analysis enables the most probable value to be determined by the mean value and the uncertainty to be assessed by the standard deviation. The random errors are generally not correlated and the probability distribution of the measured value is usually considered to follow a normal law. The reliability of a measuring system is the quality of being able to give results concentrated around the mean value. The reliability error $\delta_{r l}$ is then considered as the standard deviation. In order to be precise, a sensor must be both accurate (low systematic error) and reliable (low random error).

The global precision error of a sensor, $\delta_{p}$, is a function of the systematic error $\delta_{s}$ and the different random errors: linearity $\delta_{r}$, resolution $\delta_{r}$, quantification $\delta_{q}$, hysteresis $\delta_{h}$, and reliability $\delta_{r l}$. Thus, the global experimental uncertainty can be computed using Equation 17:

where

$$
U_{\phi_{p}}=\sqrt{U_{\phi_{l}}^{2}+U_{\phi_{r}}^{2}+U_{\phi_{q}}^{2}+U_{\phi_{h}}^{2}+U_{\phi_{r l}}^{2}+U_{\phi_{s}}}
$$

$$
U_{\phi_{r}}=\sqrt{U_{\phi_{m}}^{2}+U_{\phi_{r d}}^{2}}
$$

When the comparison between numerical and experimental values is made on a quantity which is directly measured, the experimental uncertainty is simply given by the measurement precision, possibly with a safety factor $k$ (Equation 19).

$$
U_{\phi_{E X P}}=k \delta_{p}
$$


In some other cases, the experimental value is determined by a combination of several measurements. When the experimental result is computed with an algebraic formula, the experimental uncertainty is obtained by a combination of each measurement uncertainty: absolute uncertainties are added for additive quantities and relative uncertainties are added for multiplicative quantities. When the value to be compared is determined by the mean value obtained after $N$ measurements, the uncertainty in the mean may be computed with Equation 19, where $k$ is a coverage factor determined with the Student's $t$-distribution, or Student's distribution [29]: $k=t_{\alpha(\alpha, N-1)}$, with $\alpha$ the desired level of confidence.

\subsection{Validation}

When the numerical uncertainty is estimated with Equation 4 (verification procedure), and the experimental uncertainty is determined with Equations 17-19, under certain conditions it is possible to estimate the modelling uncertainty. In particular, the modelling error can be computed with Equation 20, which combines Equations 1-2 and 16.

$$
\delta_{\phi_{\text {mod }}}=\left(\phi_{C F D}-\phi_{E X P}\right)-\left(\delta_{\phi_{n u m}}-\delta_{\phi_{E X P}}\right)
$$

However, in general $\delta_{\phi_{\text {num }}}$ and $\delta_{\phi_{E X P}}$ are not correlated and, therefore, it is convenient to re-write Equation 20 in terms of uncertainties. In particular, the numerical and the experimental uncertainties can be combined in a validation uncertainty, $U_{\phi_{v a l}}$, as shown in Equation 21:

$$
U_{\phi_{v a l}}=\sqrt{U_{\phi_{\text {num }}{ }^{2}+U_{\phi_{E X P}}^{2}}}
$$

Then, the following cases should be considered:

If $\left|\phi_{C F D}-\phi_{E X P}\right| \leq U_{\phi_{v a l}}$

then the simulation is validated at a level of $\boldsymbol{U}_{\boldsymbol{\phi}_{\boldsymbol{v} a l}}$ and $\delta_{\phi_{m o d}}$ cannot be assessed.

If $\left|\phi_{C F D}-\phi_{E X P}\right|>U_{\phi_{v a l}}$

then the simulation is non-validated and $\delta_{\phi_{m o d}}$ has the sign of $\phi_{C F D}-\phi_{E X P}$.

If $\left|\phi_{C F D}-\phi_{E X P}\right| \gg U_{\phi_{v a l}}$

then the simulation is non-validated and $\delta_{\phi_{\bmod }} \approx \phi_{C F D}-\phi_{E X P}$.

It should be noted that when the simulation is validated, the V\&V procedure does not provide any information about the modelling uncertainty which, therefore, could be much larger than expected. In addition, a simulation where $\left|\phi_{C F D}-\phi_{E X P}\right|$ is very large can be validated if the validation uncertainty is even larger. In conclusion, the 
validation of the simulation is a necessary condition, but not a sufficient condition, to assess its quality. The best use of the V\&V procedure is not only to perform a check of the simulation quality, but, importantly, it can suggest how to improve the simulation. Furthermore, when simulations are performed to identify a ranking between different sail designs, then the $V \& V$ procedure will provide indications on the level of confidence on the achieved ranking. This important aspect will be discussed in detail in Section 4.5.

\section{Example of V\&V Analysis}

\subsection{Experimental Benchmark and Numerical Setup}

The wind tunnel tests performed by [30] were modelled. The authors measured the pressure distributions on rigid model-scale sails in upwind conditions with the aim of providing an experimental benchmark for numerical simulations. The geometries and the measured pressure distributions are available from ignazioviola.com. Wind transparent wires supported the upstream sail, namely the foresail, and the downstream sail, namely the mainsail; the rigging and the hull were not present. The foot of the foresail was at $600 \mathrm{~mm}$ from the wind tunnel floor in order to experience a uniform flow outside the floor boundary layer. Figure 3 shows the test section where the open jet wind tunnel section is on the left end, the horizontal boundaries are the test section roof and floor, a distance $h=3500 \mathrm{~mm}$ apart, while open walls were used on the side. A flat plate touching the foot of the foresail was mounted under both sails. The Reynolds number was $R e=2.3 \cdot 10^{5}$. Pressures were measured on four sail sections on each sail (from Section 1 to Section 4 in Figure 3).

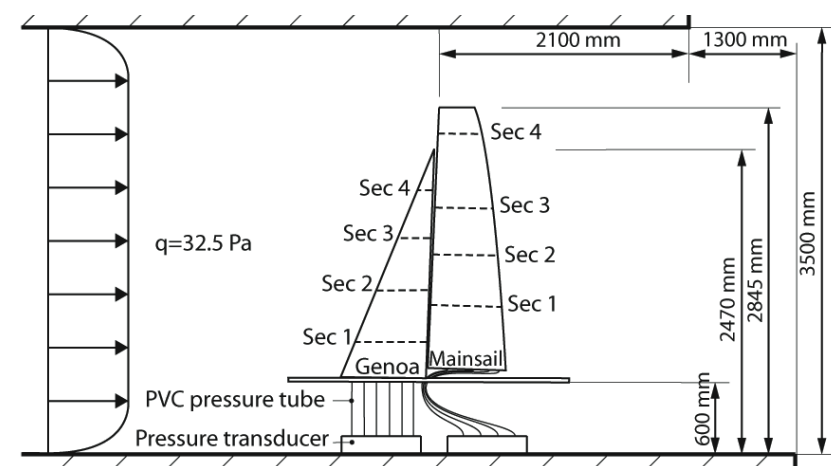

Figure 3: Experimental setup (edited from [30]).

The wind tunnel tests were modelled by solving the steady 3D RANS equations with the $k-\omega$ SST turbulence model implemented in Ansys Fluent 12.0.1. A SIMPLEC scheme, coupling velocity and pressure, and second order accuracy discretisation algorithms were used. A reference grid of 1.48 million hexahedral cells was used. On the suction (leeward) side of the sails, where regions with separated flow occur, $y^{+}<3$ was used. On the pressure (windward) side, where the boundary layer is always attached, $5<y^{+}<25$ was used. One finer grid, and three coarser grids were used to investigate the grid sensitivity, as explained in Section 3.2. Figure 4 shows a horizontal and a vertical plane of the reference grid around both rendered sails. The computational domain represents the open jet wind tunnel test section and the surrounding volume. The domain is $1.3 h$ high, $7.5 h$ long and $12 h$ wide, with $h=2.3$ $\mathrm{m}$, the height of the sails model. Non slip boundary condition was used on the sails, 
while slip condition was used to model the roof and the flat plate touching the foot of the foresail, velocity inlet on the upstream boundary (1.6h upstream of the model) and outlet on the other boundaries.

The analysis of the grid, convergence and round-off uncertainties for this simulation are presented in Sections 3.2, 3.3 and 3.4, respectively. Section 3.5 presents the validation of the computed pressure distributions.
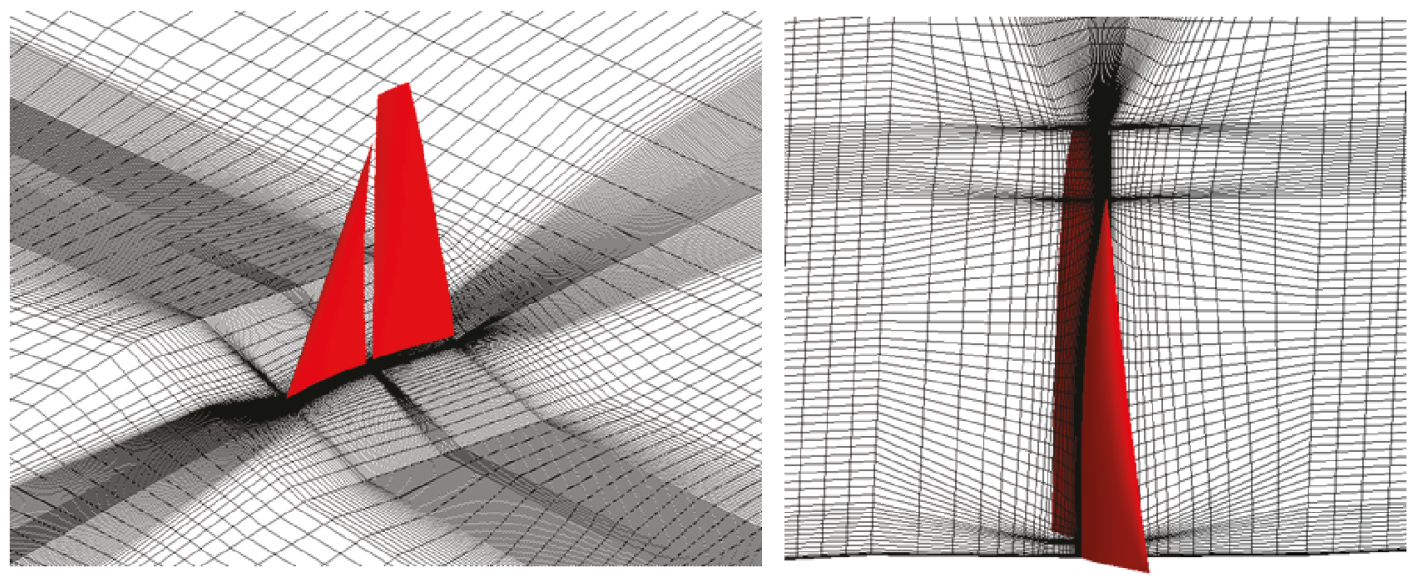

Figure 4: Horizontal and vertical grid planes of the reference grid.

\subsection{Discretisation Uncertainty}

A grid convergence study was carried out with a ratio between the distances of the grid nodes of $\sqrt[3]{2}$. Five grids with $3.19 \mathrm{M}, 1.48 \mathrm{M}$ (base), $0.84 \mathrm{M}, 0.46 \mathrm{M}$ and $0.28 \mathrm{M}$ cells were performed. The relative step ratio of the $i$-th grid is $h_{i}=(\sqrt[3]{2})^{i}$, with $i=-1,0, \ldots, 3$, resulting in $h=0.79,1.00$ (base), 1.26, 1.59, 2.00 respectively.

Figure 5 shows $\phi=C_{L} / C_{L_{\text {base }}}$ computed for different grids. The five grids do not show a convergent trend. The small differences between the lift coefficients and the absence of a trend for the relative step size suggest that the scatter is significantly larger than the differences due to the grid convergence. Therefore, the best estimation of $\phi$ is the mean value across the different simulations which, by coincidence, gives a result almost equal to $C_{L_{b a s e}}$. In fact, compared to the mean value, $\phi$ computed with the finest grid has a smaller grid convergence error but a larger scatter error, which, in this case, is the dominant error. The uncertainty for each sample can be computed with Equation 8. For every grid, $U_{C_{L_{b a s e}}}=0.92 \% C_{L_{\text {base }}}$. The uncertainty for the mean is computed with Equation 10: $U_{C_{L_{\text {mean }}}}=0.23 \% C_{L_{\text {base }}}$.

Equation 8 allows a reasonable estimate of the uncertainty when a clear grid convergence is not visible. In such a case, some authors (for instance, [9-14]) do not provide a formulation to estimate the uncertainty, while other authors (for instance, [15-18]) provide more sophisticated formulations than Equation 8. For instance, in [17] the uncertainty is computed as the minimum between the uncertainty based on the $L_{\infty}$ norm of the computed value, and the uncertainty based on the fit with a second order polynomial. For the present case, the uncertainty based on the $L_{\infty}$ norm is smaller and $U_{C_{L_{b a s e}}}=0.63 \% C_{L_{\text {base }}}$. Therefore, the use of Equation 8 leads to a more conservative estimate for $U_{C_{L_{b} a s e}}$. 


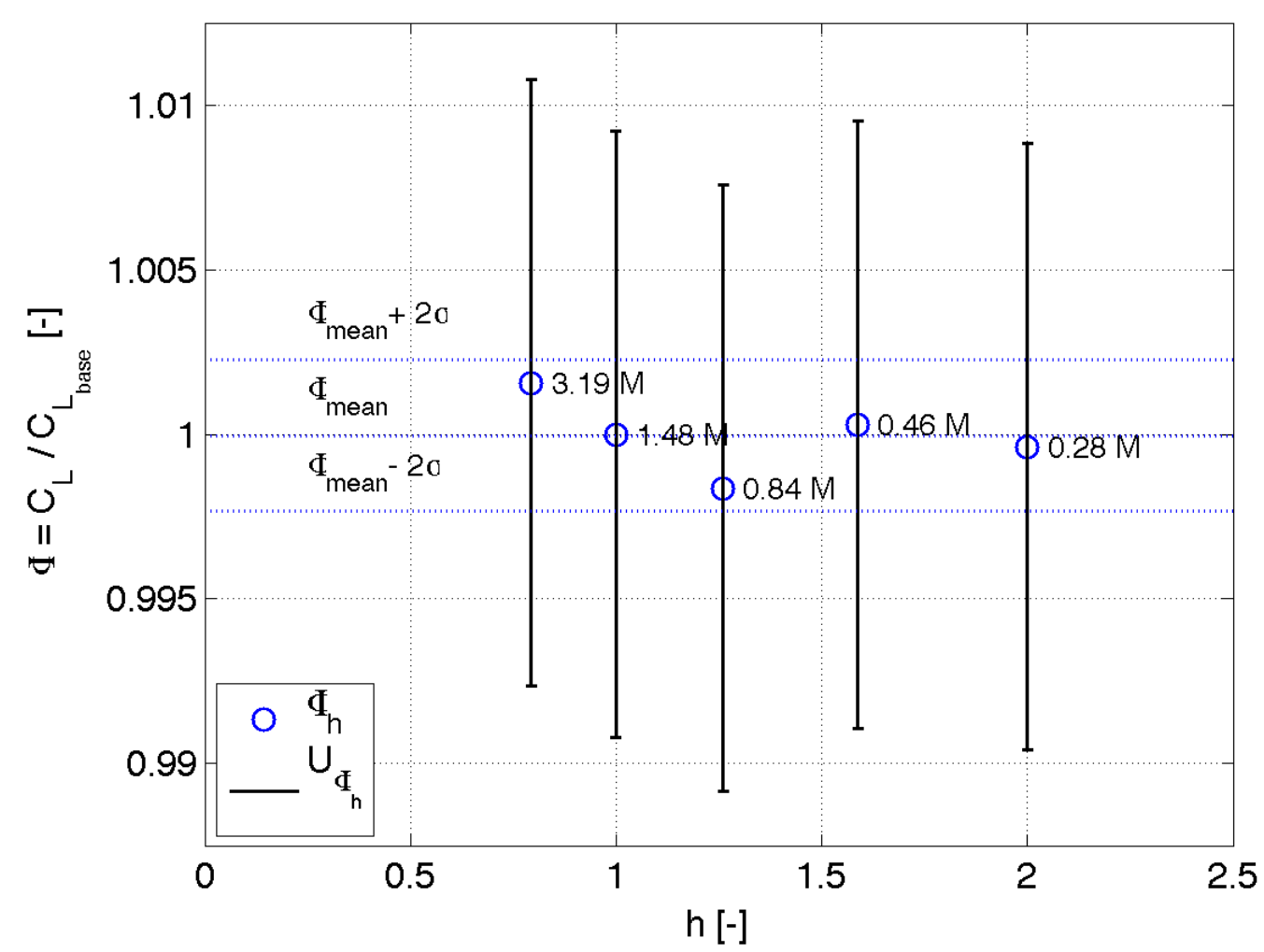

Figure 5: $C_{L}$ versus the relative grid size. Error bars show the grid uncertainty for each grid, while the dotted lines show the $C_{L}$ mean value and its uncertainty range.

Figure 6 shows $\phi=C_{D} / C_{D_{\text {base }}}$ computed for different grids. A converging trend is observed and the fit with Equation 5 allows computation of the extrapolated value $\phi_{0}=0.99$, the order of convergence $p=2.24$ and the standard deviation of the fit error $\sigma=1.8 \cdot 10^{-4}$. The uncertainty, $U_{C_{D_{\text {base }}}}=1.5 \% C_{D_{\text {base }}}$, is computed using Equation 6.

In the proposed $V \& V$ procedure, Equation 6 is used for any $p>0.95$. Conversely, other authors who assumed a theoretical order of convergence of 2, such as [17], recommend using two different equations for $0.95 \leq p \leq 2.05$ and $p>2.05$. In the present case, the procedure suggested by [17] seems to over-estimate $U_{C_{D_{\text {base }}}}\left(4.1 \% C_{D_{\text {base }}}\right)$, which results almost three times larger than the uncertainty computed with Equation 6. 


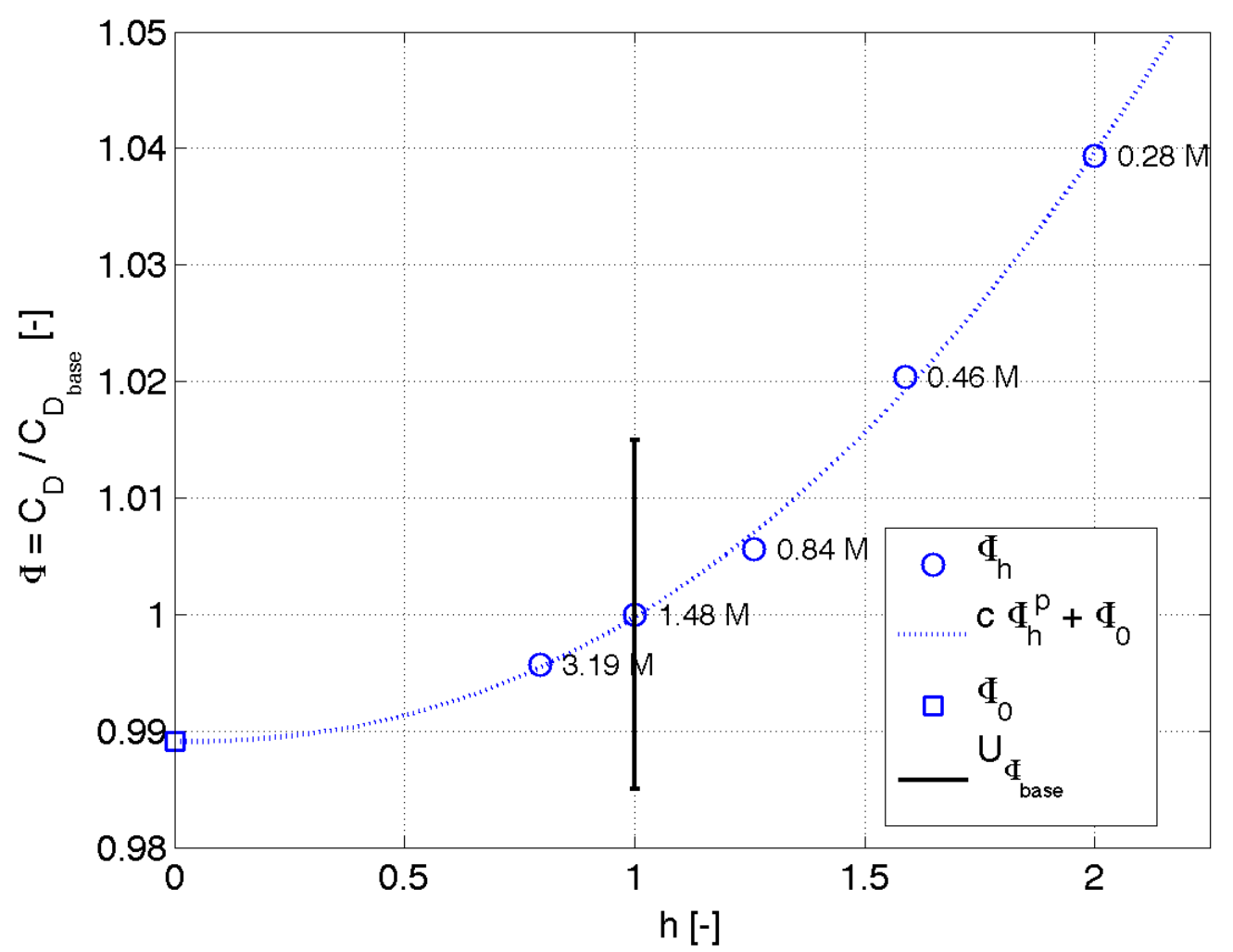

Figure 6: $C_{D}$ versus the relative grid size.

Figure 7 shows the pressure coefficient $C p=\left(p-p_{\infty}\right) / q_{\infty}$ (where $p$ is the local static surface pressure, $p_{\infty}$ is the far-field static pressure and $q_{\infty}$ is the far-field dynamic pressure) on the highest measuring section of the foresail (Section 3 in Figure 3). This is a horizontal section at $75 \%$ of the foresail height from the bottom. $C_{p}$ is plotted versus the non-dimensional chord length $x / c$ for the five different grids. $C_{p}$ computed on the surface grid nodes is fitted with a spline curve and then interpolated where the surface pressures were measured. Data fitting and interpolation introduce additional uncertainties. The standard deviation of the fitting should be significantly smaller than the other uncertainties in order to be neglected, such as in this case.

Figure 7 shows that the differences between $C_{p}$ computed with different grids are larger in the leeward region near the leading edge and, particularly, near the suction peak. 


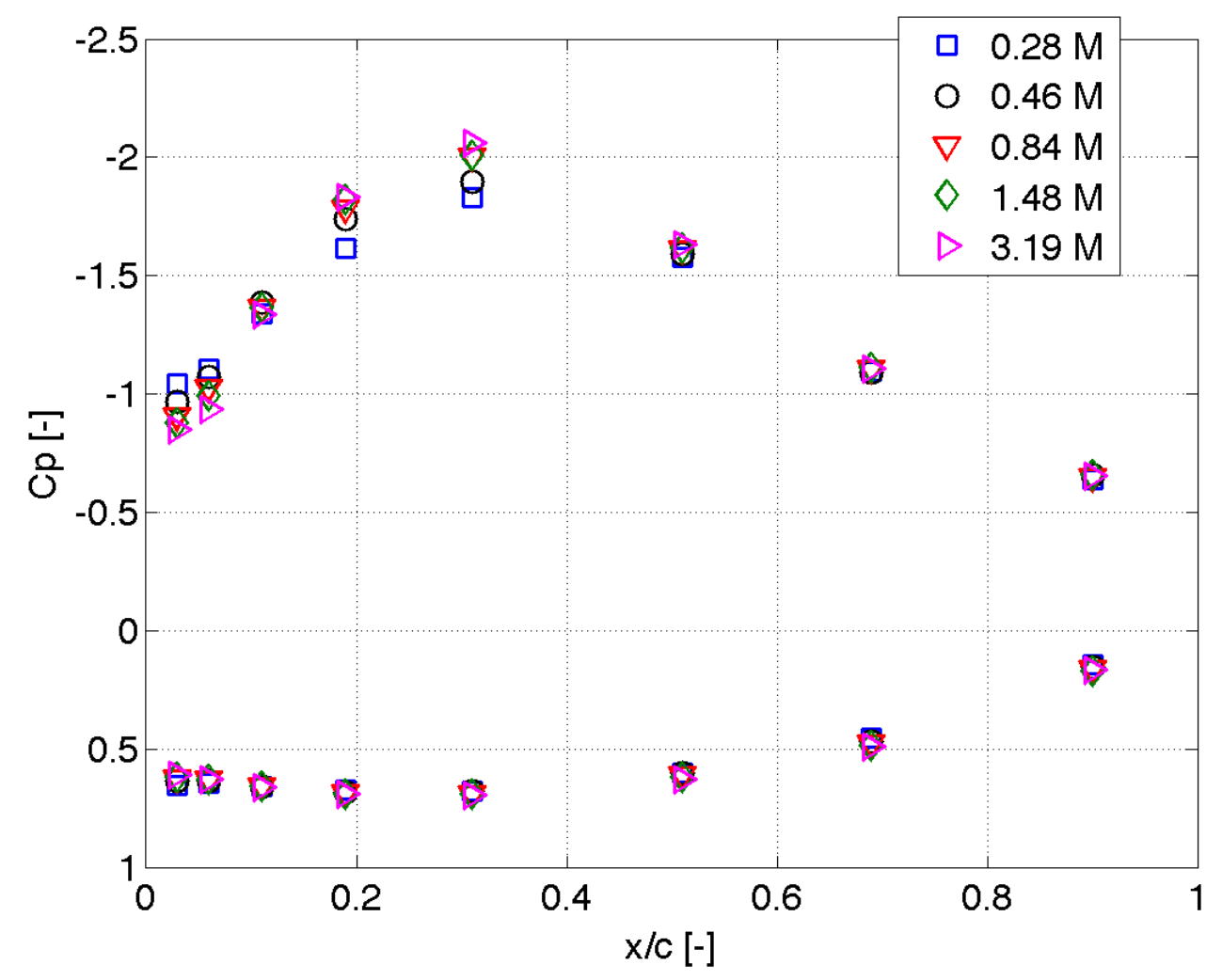

Figure 7: $C_{p}$ on Section 3 of the foresail for different grid resolutions.

The $L_{2}$ norm of the pressure coefficient along both the windward and leeward sides can be used to provide a global measurement of the pressure uncertainty:

$$
\left\|C_{p}\right\|_{L_{2}}=\sqrt{\sum_{i} C_{p_{i}}{ }^{2}}
$$

where $i$ is the index of the grid node along the plate surface.

Figure 8 shows the $L_{2}$ norm of $C_{p}$ computed with different domain sizes. A monotonic convergence is observed and $p=3.41$, therefore, Equation 6 is used to compute the relative uncertainty $U_{C_{p_{\text {base }}}}=0.7 \%\left\|C_{p_{\text {base }}}\right\|_{L_{2}}$. As mentioned above in Section 2.1, when a high order of convergence is found $(p>3)$, this should be verified with more than three grids. In fact, the high order of convergence leads to small convergence errors and thus to small uncertainties. Therefore, the uncertainty due to the scatter must be accurately estimated. Other V\&V procedures such as [17], which assume a theoretical order of convergence of 2 and therefore different formulations for $p>2.05$, lead to larger estimates of the uncertainty. For example, similarly to the estimate of $U_{C_{D_{\text {base }}}}$, the estimate for $U_{C_{p_{\text {base }}}}\left(4.1 \%\left\|C_{p_{\text {base }}}\right\|_{L_{2}}\right)$ with the formulation proposed by [17] results almost six times larger than the uncertainty computed with Equation 6. 


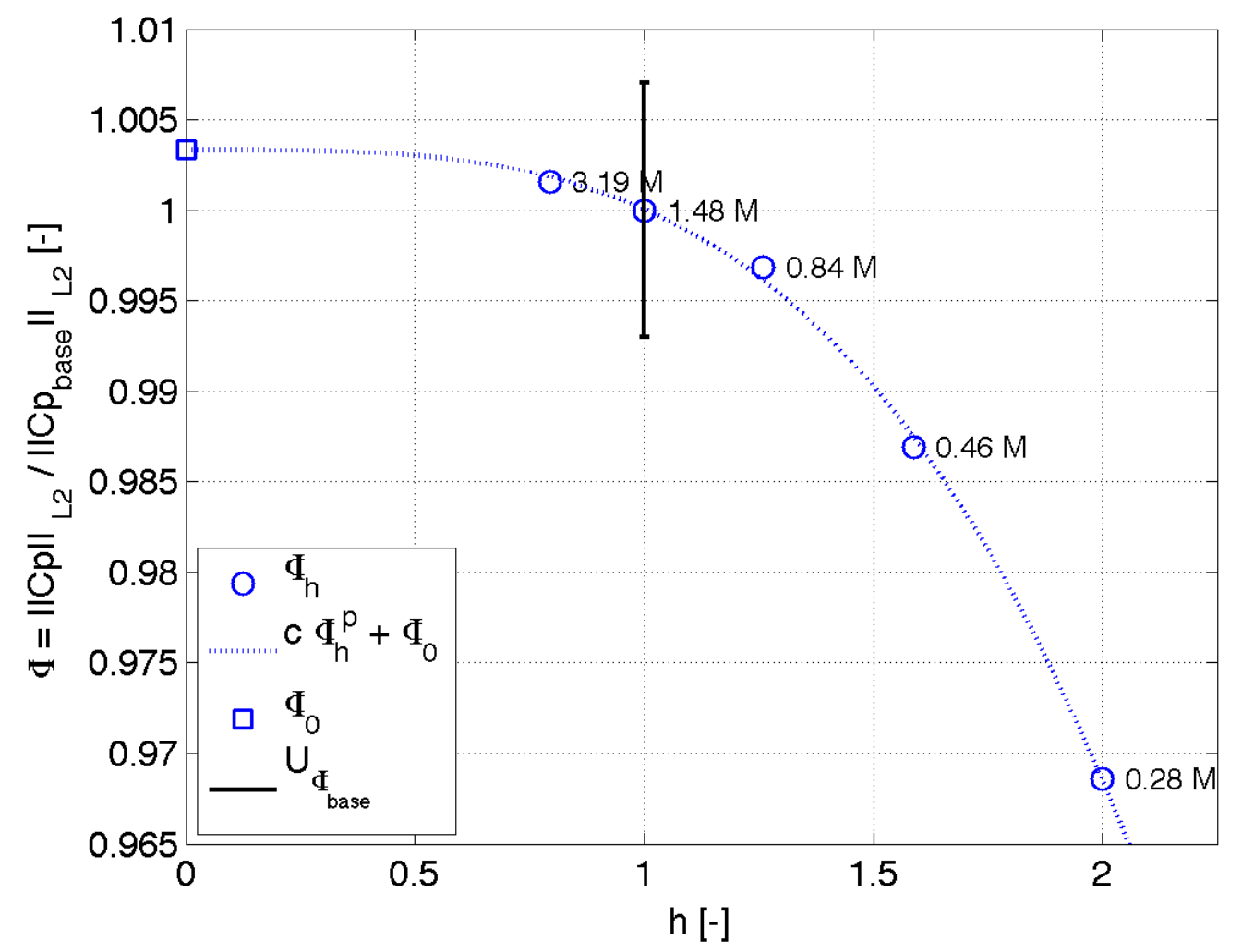

Figure 8: $L_{2}$ norm of the $C_{p}$ on Section 3 of the foresail versus the relative grid size.

Being interested only in the time-averaged solutions, the steady RANS equations were solved. If time-dependent equations were solved, then a similar analysis should be performed to assess the uncertainty due to the time discretisation (for instance, [31]).

\subsection{Convergence Uncertainty}

From the experience of the authors, who have mainly focused on the computation of aerodynamic forces and pressure distributions, the uncertainty due to the convergence is not always negligible and should be carefully assessed. In these simulations, 500 iterations were performed initially and further iterations were then performed until the variation of $U_{\phi_{c}}$ over the last 1000 iterations became smaller than $0.1 \%$ :

$$
\left\|U_{\phi_{c}}\right\|_{L_{\infty}}^{(1000 i t)}<10^{-3} \phi
$$

After every 100 iterations, $\phi_{\infty}(n)$ (Equation 11) and $U_{\phi_{\infty}}(n)$ (Equation 12) were computed and the convergence criterion (Equation 23) was checked for the lift and drag coefficients and the maximum and minimum pressures on the sail surfaces. For instance, Figure 9 shows the convergence of the lift coefficient $C_{L}$. After 2800 iterations, the convergence criterion was satisfied and the simulation was interrupted. The convergence uncertainties of the extrapolated lift coefficients (indicated by $\mathrm{x}$ ) 
that do not satisfy the convergence criterion are shown in red, while the fit curve and the convergence uncertainty $\left(U_{C_{L}}=0.08 \% C_{L_{C F D}}\right)$ of the final extrapolated lift coefficient (squared mark) is shown in blue.

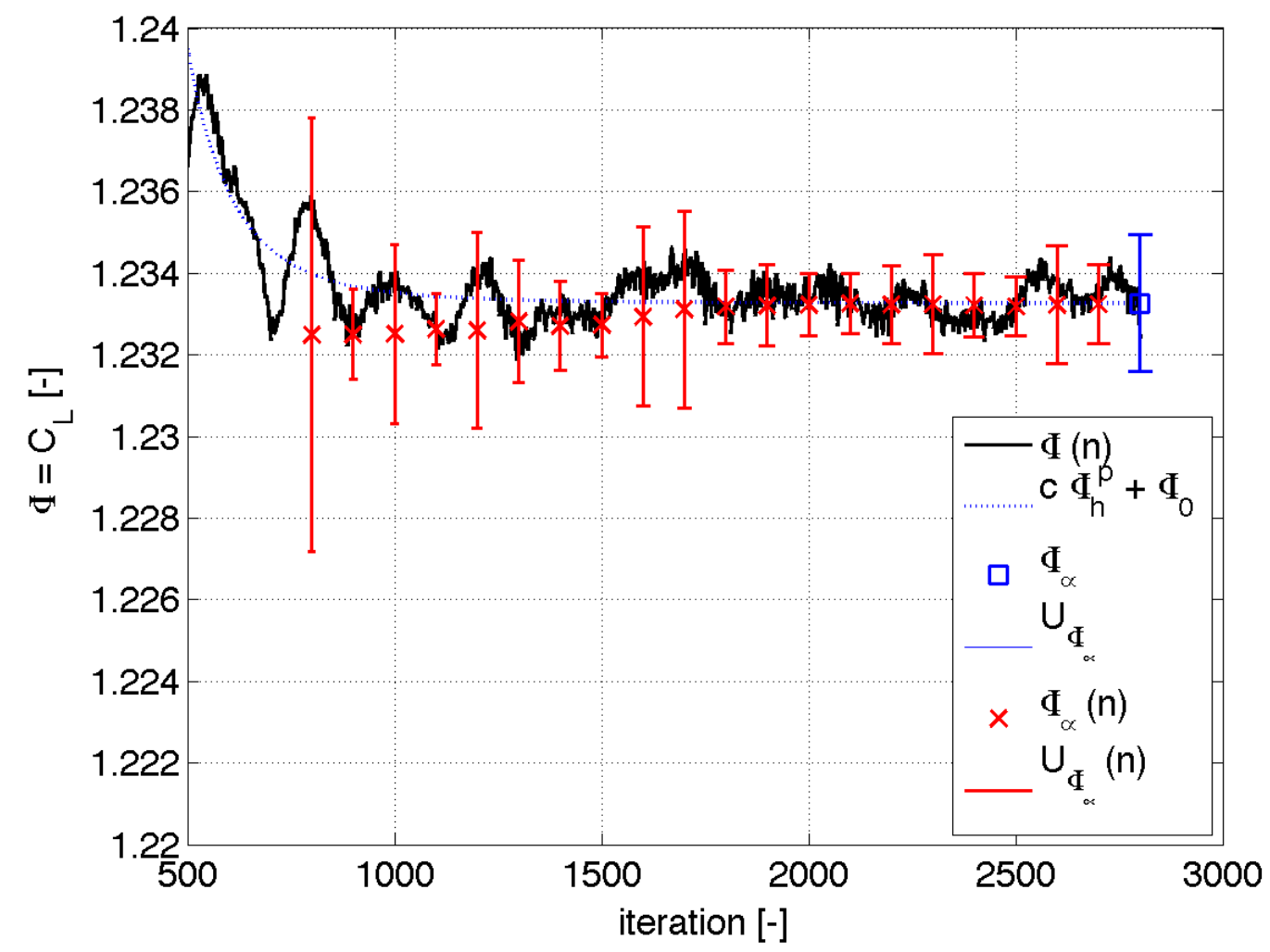

Figure 9: Convergence of $C_{L}$ and assessment of the convergence criterion.

\subsection{Round-Off Uncertainty}

The round-off uncertainty was assessed by performing 1000 further iterations both in single precision and in double precision. The data was averaged over 1000 iterations and the mean values were compared. The round-off uncertainty for the lift coefficient was $U_{C_{L}}=0.05 \% C_{L_{C F D}}$, which was computed using Equation 14.

\subsection{Validation}

Local $C_{p}$ were validated on Section 3 of the foresail. Figure 10 shows the numerical and experimental local $C_{p}$ and their uncertainties. The experimental uncertainty due to the measurement systems is $\pm 0.5 \mathrm{~Pa}$ for the surface pressure measurements and \pm 1 $\mathrm{Pa}$ for the dynamic pressure, resulting in uncertainties for the $C_{p}$ from $U_{E X P}=$ $3.2 \% C_{p}$ when $C_{p}=2$ to $U_{E X P}=4.3 \% C_{p}$ when $C_{p}=0.5$. Several measurements were repeated in order to estimate the uncertainty due to the re-positioning of the sails. This uncertainty is substantial in the region near the leading edge on both sides of the sail. The experimental uncertainty presented herein takes into account the uncertainty due to the measurement systems and the maximum difference between repeated measurements multiplied by a safety factor of 1.5.

In this simulation, the uncertainties due to the grid resolution and the convergence were more than two orders of magnitude larger than the uncertainty due to the round 
off which, therefore, was neglected. For each known experimental $C_{p}$, the validation uncertainty was computed using Equation 21 and the validation was performed for the base grid. Tables 1 and 2 show the results of the validation for each pressure tap. The validation allows the identification of an unexpected but interesting modelling error. In fact, while the differences between the computed and measured $C_{p}$ are small compared with their uncertainties on the leeward side, meaning that the "noise" in the numerical computation and in the experimental measurement does not allow estimation of the modelling error, on the windward side the noise level is smaller and the differences are larger. In particular, the computed pressure is closer to the stagnation pressure than the measured pressure. This is possibly due to a modelling error, i.e. a difference between the CFD model and the experimental model. In fact, while the numerical model of the sail has zero thickness, the thickness of the experimental model is about $1 \%$ of the sail chord and the sails were chamfered at about $20^{\circ}$ on the windward side to produce a sharp leading edge. Figure 11 shows a schematic diagram of the leading edges of the CFD and the experimental models. In the experimental setup, between the stagnation point and the first pressure tap there is the sharp angle of the chamfer, which causes the flow to accelerate and the pressure to drop.

It is interesting to note that if a coarse grid were used, the largest difference between the numerical and the experimental $C_{p}$ would be on the leading edge on the leeward side (compare Figures 7 and 10). However, the V\&V procedure shows that while the numerical/ experimental difference decreases on the leeward side, on the windward side the difference remains constant. Therefore, it shows that the side where the CFD model does not represent the experimental model is the windward side and not the leeward side.

On each of the eight measuring sections, the $L_{2}$ norms of the numerical and experimental $C_{p}$ were computed. The discretisation, convergence and experimental uncertainties were achieved by computing the $L_{2}$ norm of the uncertainties along the sail section. The validation uncertainty was computed using Equation 21 and validation was assessed. Table 3 shows that the $L_{2}$ norm of $C_{p}$ was validated at levels of up to $30 \%$ for all of the measured sections. 


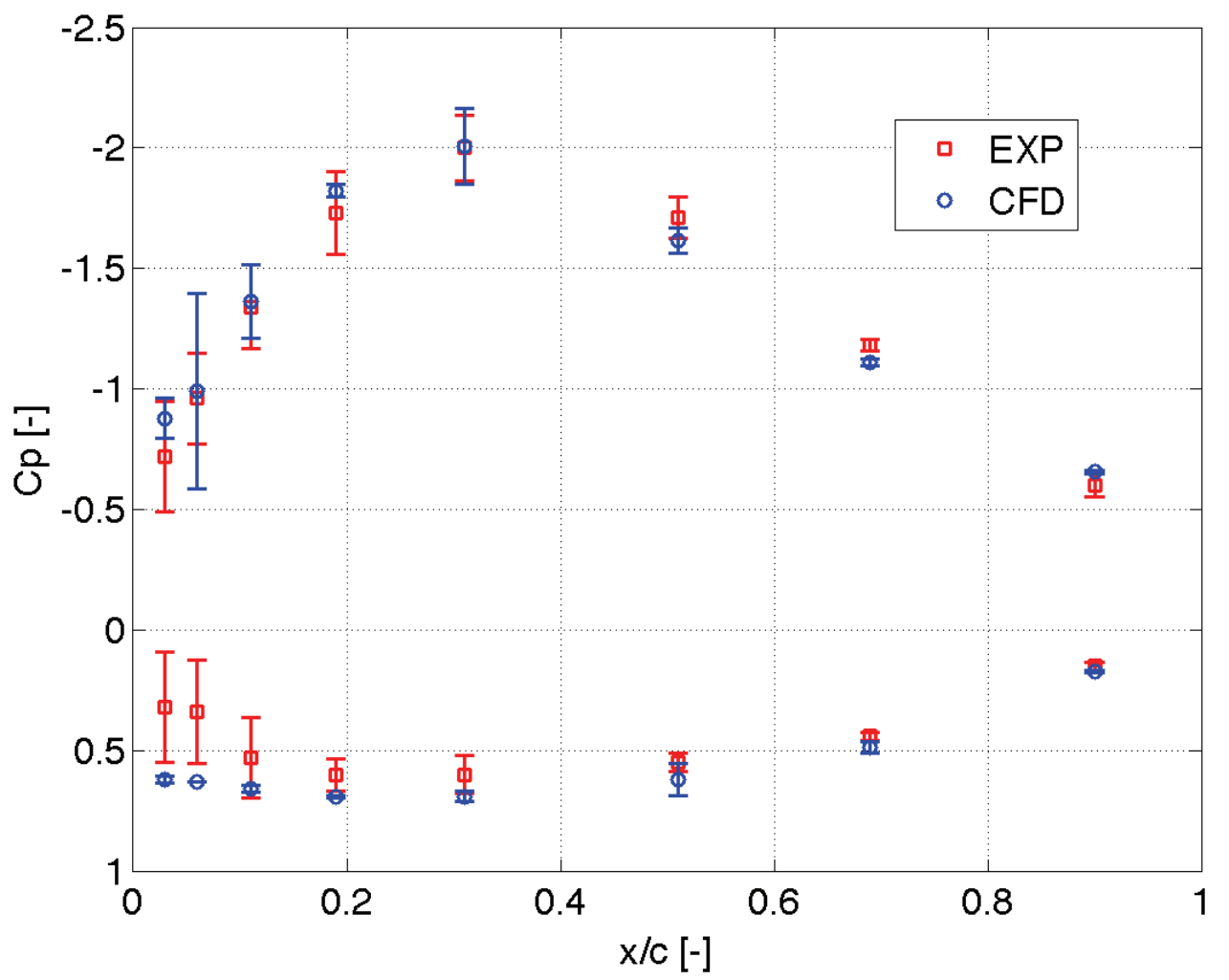

Figure 10: Measured and computed distributions of $C_{p}$ on Section 3 of the foresail. Error bars show the experimental and the grid uncertainties.

NUMERICAL MODEL

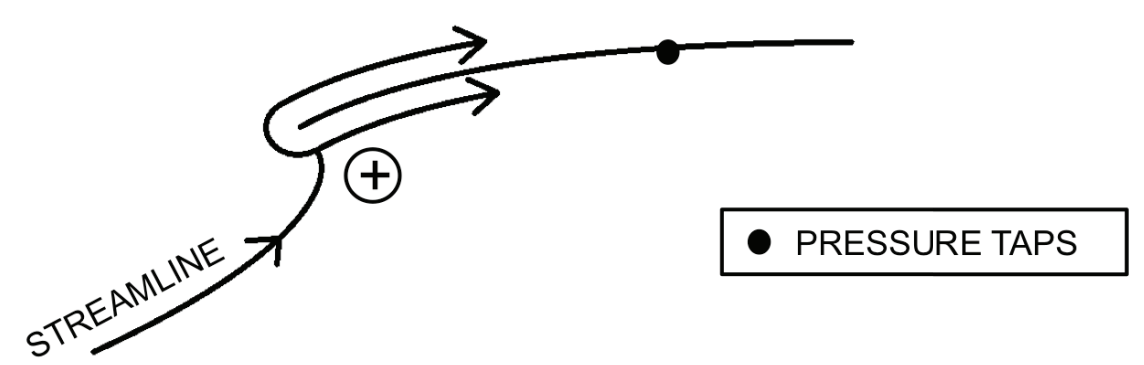

EXPERIMENTAL MODEL

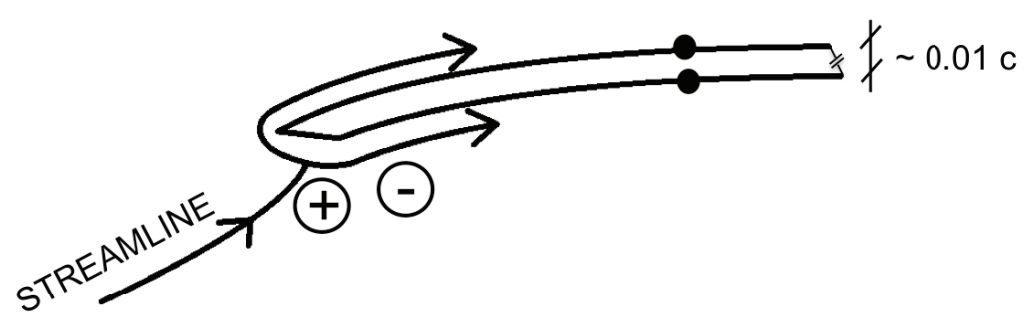

Figure 11: Schematic drawing of the sail's leading edge for the numerical and experimental models. 


\section{Ranking}

It is common practice to assume that the differences between the CFD results and the experimental data are almost constant across similar geometries and, therefore, that the relative rankings between these geometries are correctly computed by CFD despite the absolute error. This argument is often used to justify the use of low grid resolutions. In the experience of the authors this is a very dangerous assumption, which may be incorrect. In this Section, it is shown that the uncertainty analysis can be used to compute the probability that the ranking is correct.

A 2D simulation of the horizontal section at the height of Section 3 of the foresail was performed, where the same conditions and numerical setup used in the 3D simulations presented above (Section 3.1) were used. The camber of the foresail section was $13 \%$ of its chord. Four different geometries were achieved when stretching the sail section in the direction perpendicular to the chord, leading to different cambers, while the chord was kept constant. Thus, five different foresail sections were tested with cambers that were $13 \%, 16.5 \%, 20 \%, 23.5 \%$ and $27 \%$ of the chord, respectively. For each geometry, three different grid resolutions were used with relative step sizes $h=1.26,1.00$ and 0.50 .

The five geometries were ranked based on the drive force coefficient $C_{x}$, which is the aerodynamic force projection towards the sailed course divided by the dynamic pressure and the length of the two sail sections. Figure 5 shows the five different foresail sections and the mainsail section, which was unchanged for the five tested configurations.

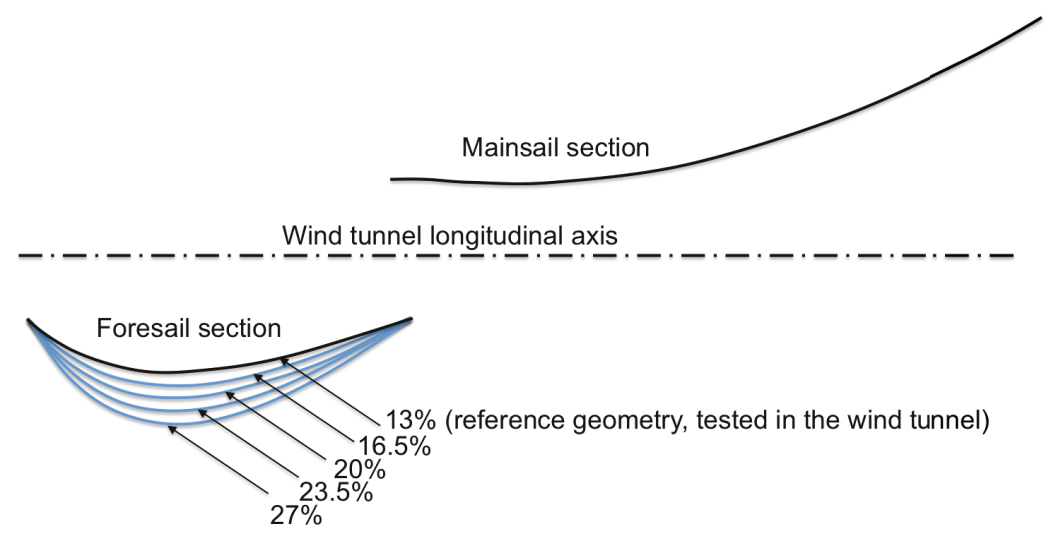

Figure 12: Five different geometries tested.

Figure 13 shows $C_{x}$ versus the camber/ chord ratio for the three grids. Error bars are presented for the base grid (solid line). The uncertainties for the three smallest cambers $\left(0.015 \leq U_{C_{x}} \leq 0.027\right)$ are of one order of magnitude smaller than the uncertainties for the two largest cambers $\left(0.453 \leq U_{C_{x}} \leq 0.5070\right)$, which are not presented in Figure 3 because they are off the scale. 


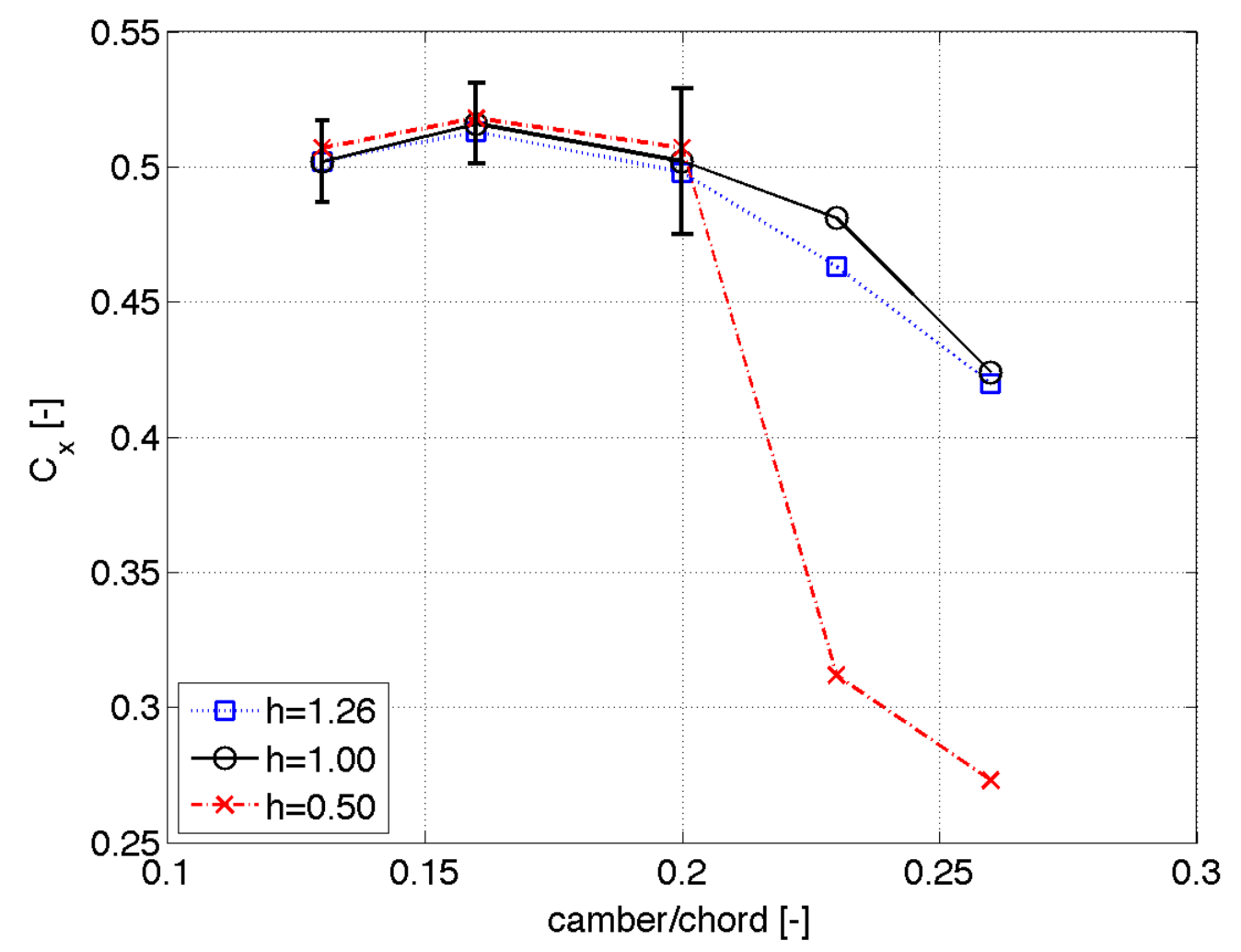

Figure 13: $C_{x}$ of 2D sail sections versus the camber/ chord ratio of the aft sail for three grid resolutions.

For the three smallest cambers, the higher the grid resolution, the lower the shear stress and, therefore, the higher $C_{x}$. When the camber increases, $C_{x}$ increases due to the larger lift allowed by the more cambered profile. However, increasing the camber further leads $C_{x}$ to decrease due to the larger region affected by trailing edge separation. The higher the grid resolution, the more upstream is the trailing edge separation point. Therefore, while high grid resolution predicts higher $C_{x}$ for small cambers, for the largest two cambers it predicts a larger $C_{x}$ reduction. The uncertainty increase is due to a jump upstream of the trailing edge separation point when the grid resolution increases.

For each couple of successive cambers $(13 \%-16.5 \% ; 16.5 \%-20 \%$, etc.) the probability that one of the two geometries allows a higher $C_{x}$ than the other one can be computed. For instance, $C_{x}$ is $3 \%$ higher for the $16.5 \%$ camber than for the $13 \%$ camber. The uncertainty of this difference is given by Equation 24:

$$
U_{(16.5 \%-13 \%)}=\sqrt{U_{13 \%}{ }^{2}+U_{16.5 \%}{ }^{2}}=4.2 \%
$$

The uncertainty of the difference is larger than the difference itself and, therefore, the probability that the ranking between the two is correct is lower than $95 \%$. In particular, the integral from 0 to $\infty$ of a normal distribution with mean value $3 \%$ and standard deviation $2.1 \%$, which is equal to half of the uncertainty, is 0.91 . Therefore, there is a $91 \%$ probability that the ranking is correct. The same analysis can be performed for 
the successive three couples of differences $(16.5 \%-20 \%, 20 \%-23.5 \%$, $23.5 \%-26 \%$ ), leading to $82 \%, 53 \%$ and $56 \%$ probabilities, respectively, that the computed rankings are correct ${ }^{1}$. Therefore, the uncertainty analysis allows distinction between the highly probable rankings of the first two couples of cambers, and the very uncertain rankings of the last two couples of cambers. It is interesting to note that if only one grid was computed then the larger differences between the last two couples of cambers compared with the first two couples would have led to the opposite conclusion.

\section{Conclusions}

Verification and validation of numerical simulations is still rarely performed in sail aerodynamics, while its use allows quality of the simulations to be enhanced and allows a more conscious use of its results. Unfortunately it can be complicated and time consuming. In recent years three different procedures have been proposed. However, while one of them does not consider the existence of scatter in the results, which can be significant in sail aerodynamics, the other two require the knowledge of the theoretical order of convergence, which is often unknown when commercial codes are used.

In the present paper a simplified verification and validation procedure is proposed with the aim of providing a simple reference for sail aerodynamicists. Examples of results are also provided in order to show the possible outcomes and uses of the procedure.

In particular, the grid uncertainty for the aerodynamic lift, drag and pressure distributions for two sails in upwind conditions are presented. The computed local surface pressures are validated against experimental measurements, showing that the verification and validation procedure may allow identification of modelling errors, such as the neglected sail thickness. It is also shown that global aerodynamic parameters such as the lift, drag and $L 2$ norm of the pressure coefficient can be computed with uncertainties of the order of $1 \%$, with a number of cells of the order of one million.

An example of convergence uncertainty and round-off uncertainty is provided, showing that these uncertainties can be several orders of magnitude smaller than the grid uncertainty.

Finally, the necessity of considering the uncertainty when different geometries or conditions are ranked is presented. In particular, it was shown how the probability that the ranking is correct can be estimated knowing the uncertainty in the computation of the value used to rank.

\footnotetext{
${ }^{1}$ A probability of $50 \%$ in the ranking means that there is the same probability that the ranking is correct and incorrect.
} 


\section{References}

1. Consejo Superior de Cameras de Commercio (CSCC), 2007. Impacto Economico de la 32 a America's Cup, Madrid.

2. Ljungqvist, K., Axfors, B., Tunander, H., Orych, M., Vesting, F. Larsson, L., 2012. Investigation of Keel Bulbs for Sailing Yachts, Proc. $4^{\text {th }}$ High Perf. Yacht Design Conf. (HPYD4), 12-13 March, Auckland.

3. Larsson, L., Stern, F., Bertram, V., 2002. Gothenburg 2000 - A Workshop on Numerical Hydrodynamics, Department of Naval Architecture and Ocean Engineering, Chalmers University of Technology, Gothenburg.

4. Larsson, L., Stern, F., Visonneau, M., 2010. Proceedings of the Workshop on Numerical Hydrodynamics, Gothenburg, Sweden, 2010.

5. Hino, T., 2005. Proceedings of the CFD Workshop, Tokyo, NMRI report.

6. Eça, L., Hoekstra, M., 2004. Proceedings of the Workshop on CFD Uncertainty Analysis, October, Lisbon.

7. Eça, L., Hoekstra, M., 2006a. Proceedings of the $2^{\text {nd }}$ Workshop on CFD Uncertainty Analysis, Instituto Superior Tecnico, Lisbon.

8. Eça, L., Hoekstra, M., 2008. Proceedings of the $3^{\text {rd }}$ Workshop on CFD Uncertainty Analysis, Instituto Superior Tecnico, Lisbon.

9. Roache, P.J., 1998. Verification of Codes and Calculations. AIAA J. 36 (5), 696702.

10. Roache P.J., 2009. Fundamentals of Verification and Validation, Albuquerque, NM Hermosa Publisher.

11. Stern, F., Wilson, R.V., Coleman, H.W., Paterson, E.G., 2001. Comprehensive Approach to Verification and Validation of CFD Simulations - Part I: Methodology and Procedures, ASME J. Fluids Eng., 123, 793-802.

12. Xing, T., Stern, F., 2008. Factors of Safety for Richardson Extrapolation for Industrial Applications, IIHR Report No. 466.

13. Xing, T. Stern, F., 2009. Factors of Safety for Richardson Extrapolation for Industrial Applications, IIHR Report No. 469.

14. Xing, T., Stern, F., 2010. Factors of Safety for Richardson Extrapolation, $J$. Fluids Eng., 132 (6), 061403-13.

15. Eça, L., Hoekstra, M., 2002. An Evaluation of Verification Procedures for CFD Applications, Proc. $24^{\text {th }}$ Symp. on Naval Hydrod., Fukuoka, Japan. 
16. Eça, L., Hoekstra, M., 2006b, Discretization Uncertainty Estimation Based on a Least Squares Version of the Grid Convergence Index, Proc. $2^{\text {nd }}$ Workshop on CFD Uncertainty Analysis, Lisbon, Portugal.

17. Eça, L., Vaz, G., Hoekstra, M., 2010a. Code Verification, Solution Verification and Validation in RANS Solvers, Proc. ASME $29^{\text {th }}$ Int. Conf. OMAE2010, Shanghai, China.

18. Eça, L., Vaz, G., Hoekstra, M., 2010b. A Verification and Validation Exercise for the Flow Over a Backward Facing Step, Proc. $5^{\text {th }}$ Eur. Conf. on Comp. Fluid Dynamics, ECCOMAS CFD 2010, Eds. Pereira J.C.F., Sequeira A., Lisbon.

19. American Society of Mechanical Engineers ASME), 2009. Standard for Verification and Validation in Computational Fluid Dynamics, ASME Committee PTC61, 2008, ASME V\&V 20-2009, Alexandria, VA.

20. International Towing Tank Conference (ITTC), 1999. Verification and Validation Methodology and Procedures (4.9-04-01-01). Recommended Procedures, Uncertainty Analysis in CFD.

21. International Towing Tank Conference (ITTC), 2002. Verification and Validation Methodology and Procedures (7.5-03-01-01). Recommended Procedures, Uncertainty Analysis in CFD.

22. International Towing Tank Conference (ITTC), 2008. Verification and Validation Methodology and Procedures (7.5-03-01-01). Recommended Procedures, Uncertainty Analysis in CFD.

23. Eça, L., Hoekstra, M., 2006. On the Influence of the Iterative Error in the Numerical Uncertainty of Ship Viscous Flow Calculations. Proc. $26^{\text {th }}$ Symp. on Naval Hydrod.. Rome.

24. Versteeg, H.K., Malalasekera, W., 1995. An Introduction to Computational Fluid Dynamics, The Finite Volume Method, Longman Scientific and Technical, Harlow, UK.

25. Richardson, L.F., 1911. The Approximate Arithmetical Solution by Finite Differences of Physical Problems Including Differential Equations, with an Application to the Stresses in a Masonry Dam. Philosophical Trans. Royal Society of London, Series A 210 (459-470), 307-357.

26. Eça, L., Hoekstra M., 2009. On the Numerical Accuracy of the Prediction of Resistance Coefficients in Ship Stern Flow Calculations. J. Mar. Sci. and Tech., 14 (1), 2-18.

27. Topping ,J., 1972. Errors of Observation and their Treatment, London, Chapman and Hall.

28. Cunningham, M.J., 1981. Measurements Errors and Instrument Inaccuracies, $J$. Phys. E. Sci. Instrum., 14, 901. 
29. Joint Committee for Guides in Metrology (JCGM), 2008. Evaluation of measurement data - Guide to the expression of uncertainty in measurement, JCGM 100-2008.

30. Viola, I.M., Pilate, J., Flay, R.G.J., 2011. Upwind Sail Aerodynamics: a Pressure Distribution Database for the Validation of Numerical Codes, Int. J. of Small Craft Tech., Trans. RINA, 153 (B1), 47-58.

31. Fathi, F. Eça, L., Borsboom, M., 2011. An example of Code Verification in the simulation of wave propagation. Proc. Int. Conf. on Offshore Mech. and Arctic Eng. (OMAE), vol. 6, pp. 313-321, 19-24 June, Rotterdam, The Netherlands. 
Table 1: Validation of local $C_{p}$ on the windward side of Section 3 of the foresail

\begin{tabular}{|l|c|c|c|c|c|c|c|c|}
\cline { 2 - 9 } \multicolumn{1}{c|}{} & \multicolumn{8}{|c|}{ Windward Side } \\
\hline$x / c$ & 0.03 & 0.06 & 0.11 & 0.19 & 0.31 & 0.51 & 0.69 & 0.90 \\
\hline \hline$C_{p_{\text {EXP }}}$ & 0.32 & 0.34 & 0.53 & 0.60 & 0.60 & 0.55 & 0.44 & 0.15 \\
\hline$C_{p_{C F D}}$ & 0.62 & 0.63 & 0.66 & 0.69 & 0.69 & 0.62 & 0.49 & 0.17 \\
\hline$C_{p_{C F D}}-C_{p_{E X P}}$ & 0.30 & 0.29 & 0.12 & 0.093 & 0.090 & 0.069 & 0.049 & 0.028 \\
\hline$U_{C_{p_{g}}}$ & 0.016 & 0.001 & 0.014 & 0.004 & 0.022 & 0.068 & 0.024 & 0.006 \\
\hline$U_{C_{p_{C}}}$ & 0.002 & 0.002 & 0.002 & 0.002 & 0.002 & 0.002 & 0.002 & 0.002 \\
\hline$U_{C_{p_{\text {num }}}}$ & 0.018 & 0.003 & 0.016 & 0.006 & 0.024 & 0.070 & 0.027 & 0.008 \\
\hline$U_{C_{p_{\text {EXP }}}}$ & 0.229 & 0.213 & 0.167 & 0.067 & 0.079 & 0.040 & 0.017 & 0.016 \\
\hline \hline$U_{C_{p_{\text {val }}}}$ & 0.229 & 0.213 & 0.168 & 0.067 & 0.083 & 0.081 & 0.032 & 0.018 \\
\hline Validated? & no & no & yes & no & no & yes & no & no \\
\hline
\end{tabular}

Table 2: Validation of local $C_{p}$ on the leeward side of Section 3 of the foresail

\begin{tabular}{|l|c|c|c|c|c|c|c|c|}
\cline { 2 - 9 } \multicolumn{1}{c|}{} & \multicolumn{7}{|c|}{ Leeward Side } \\
\hline$x / c$ & 0.03 & 0.06 & 0.11 & 0.19 & 0.31 & 0.51 & 0.69 & 0.90 \\
\hline \hline$C_{p_{E X P}}$ & -0.72 & -0.96 & -1.34 & -1.73 & -2.00 & -1.71 & -1.18 & -0.60 \\
\hline$C_{p_{C F D}}$ & -0.88 & -0.99 & -1.36 & -1.82 & -2.01 & -1.61 & -1.11 & -0.66 \\
\hline $\mid C_{p_{C F D}}-C_{p_{E X P}}$ & 0.16 & 0.03 & 0.02 & 0.09 & 0.01 & 0.09 & 0.07 & 0.05 \\
\hline$U_{C_{p_{g}}}$ & 0.082 & 0.404 & 0.153 & 0.026 & 0.155 & 0.052 & 0.015 & 0.008 \\
\hline$U_{C_{p_{C}}}$ & 0.005 & 0.005 & 0.005 & 0.005 & 0.005 & 0.005 & 0.005 & 0.005 \\
\hline$U_{C_{p_{\text {num }}}}$ & 0.087 & 0.409 & 0.158 & 0.032 & 0.161 & 0.057 & 0.021 & 0.013 \\
\hline$U_{C_{p_{\text {EXP }}}}$ & 0.230 & 0.187 & 0.174 & 0.171 & 0.134 & 0.087 & 0.024 & 0.046 \\
\hline \hline$U_{C_{p_{\text {val }}}}$ & 0.246 & 0.449 & 0.235 & 0.174 & 0.209 & 0.104 & 0.032 & 0.048 \\
\hline Validated? & yes & yes & yes & yes & yes & yes & no & no \\
\hline
\end{tabular}


Table 3. Validation of $\left\|C_{p}\right\|_{L_{2}}$ for the measuring sections of the two sails.

\begin{tabular}{|l|c|c|c|c|c|c|c|c|}
\cline { 2 - 9 } \multicolumn{1}{c|}{} & \multicolumn{4}{c|}{ Foresail } & \multicolumn{4}{c|}{ Mainsail } \\
\hline Section & 1 & 2 & 3 & 4 & 1 & 2 & 3 & 4 \\
\hline \hline$\left\|C_{p_{E X P}}\right\|$ & 4.88 & 4.07 & 4.08 & 4.35 & 2.67 & 3.09 & 3.08 & 2.95 \\
\hline$\left\|C_{p_{C F D}}\right\|$ & 5.45 & 4.45 & 4.25 & 4.31 & 2.53 & 2.86 & 2.91 & 2.93 \\
\hline$\left\|C_{p_{C F D}}\right\|-\left\|C_{p_{E X P}}\right\|$ & 0.57 & 0.38 & 0.17 & 0.04 & 0.14 & 0.23 & 0.17 & 0.02 \\
\hline$\left\|U_{C_{p_{g}}}\right\|$ & 0.064 & 0.018 & 0.007 & 0.021 & 0.028 & 0.065 & 0.15 & 0.068 \\
\hline$\left\|U_{C_{p_{C}}}\right\|$ & 0.021 & 0.021 & 0.021 & 0.021 & 0.021 & 0.021 & 0.021 & 0.021 \\
\hline$\left\|U_{C_{p_{\text {num }}} \|}\right\|$ & 0.085 & 0.039 & 0.028 & 0.042 & 0.049 & 0.086 & 0.171 & 0.089 \\
\hline$\left\|U_{C_{p_{\text {EXP }}}}\right\|$ & 0.681 & 0.703 & 0.687 & 0.66 & 0.811 & 0.811 & 0.764 & 0.687 \\
\hline \hline$\left\|U_{C_{p_{\text {val }}}}\right\|$ & 0.687 & 0.704 & 0.688 & 0.661 & 0.812 & 0.815 & 0.783 & 0.693 \\
\hline Validated? & yes & yes & yes & yes & yes & yes & yes & yes \\
\hline
\end{tabular}

